Article

\title{
Primal Lower Nice Functions in Reflexive Smooth Banach Spaces
}

\author{
Messaoud Bounkhel *iD and Mostafa Bachar \\ Department of Mathematics, College of Science, King Saud University, \\ P.O. Box 2455, Riyadh 11451, Saudi Arabia; mbachar@ksu.edu.sa \\ * Correspondence: bounkhel@ksu.edu.sa; Tel.: +966-11-467625
}

Received: 3 October 2020; Accepted: 10 November 2020; Published: 19 November 2020

check for updates

\begin{abstract}
In the present work, we extend, to the setting of reflexive smooth Banach spaces, the class of primal lower nice functions, which was proposed, for the first time, in finite dimensional spaces in [Nonlinear Anal. 1991, 17, 385-398] and enlarged to Hilbert spaces in [Trans. Am. Math. Soc. 1995, 347, 1269-1294]. Our principal target is to extend some existing characterisations of this class to our Banach space setting and to study the relationship between this concept and the generalised $V$-prox-regularity of the epigraphs in the sense proposed recently by the authors in [J. Math. Anal. Appl. 2019, 475, 699-29].
\end{abstract}

Keywords: $\quad$ generalised projection; $V$-proximal subdifferential; $V$-proximal normal cone; limiting $V$-proximal subdifferential; generalised prox-regularity

\section{Introduction and Preliminaries}

In all the paper, $X$ will denote a reflexive smooth Banach space, unless otherwise specified. We quote from [1] the definition of the $V$-proximalsubdifferential (called in [1] the analytic proximal subdifferential).

Definition 1 ([1]). Let $f: X \rightarrow \mathbb{R} \cup\{+\infty\}$ be a lower semi-continuous function (l.s.c.), and $x \in X$ with $f(x)$ is finite. We recall that the $V$-proximal subdifferential of $f$ at $x$ is defined as $x^{*} \in \partial^{\pi} f(x)$ if and only if there exists $\sigma>0$ such that:

$$
\left.\left\langle x^{*} ; x^{\prime}-x\right\rangle \leq f\left(x^{\prime}\right)-f(x)+\sigma V\left(J(x), x^{\prime}\right)\right), \forall x^{\prime} \text { near } x .
$$

Here, $J$ is the normalised duality mapping on $X$ and $V$ is the functional defined from $X^{*} \times X$ to $[0, \infty)$ by:

$$
V\left(x^{*}, x\right)=\left\|x^{*}\right\|^{2}-2\left\langle x^{*} ; x\right\rangle+\|x\|^{2}, \quad \text { for any }\left(x^{*}, x\right) \in X^{*} \times X \text {. }
$$

The $V$-proximal normal cone of a non-empty closed subset $S$ in $X$ at $x \in S$ is defined as the $V$-proximal subdifferential of the indicator function of $S$, that is $N^{\pi}(S ; x)=\partial^{\pi} \psi_{S}(x)$. The limiting $V$-proximal normal cone is defined as:

$$
N^{L \pi}(S ; \bar{x})=\limsup _{x \rightarrow \bar{x}} N^{\pi}(S ; x):=\left\{w-\lim _{n} x_{n}^{*}: x_{n}^{*} \in N^{\pi}\left(S ; x_{n}\right) \text { with } x_{n} \rightarrow^{S} \bar{x}\right\} .
$$

Another proximal subdifferential $\partial_{G}^{\pi} f(x)$ is defined geometrically, in [1], via the $V$-proximal normal cone of the epigraph as follows:

$$
\partial_{G}^{\pi} f(x)=\left\{x^{*} \in X^{*}:\left(x^{*} ;-1\right) \in N^{\pi}(\text { epi } f ;(x, f(x)))\right\},
$$


where epif $:=\{(x, r) \in X \times \mathbb{R}$ such that $f(x) \leq r\}$. It was proven in [1] that we always have $\partial^{\pi} f(x) \subset \partial_{G}^{\pi} f(x)$. In [1], $\partial_{G}^{\pi}$ was called the geometric proximal subdifferential.

Using the functional $V$, Alber in [2] introduced the generalised projection on closed convex sets $S$ as follows: $\bar{x} \in \pi_{S}\left(x^{*}\right)$ if and only if $V\left(x^{*}, \bar{x}\right)=\inf _{x \in S} V\left(x^{*}, x\right)$. In [3], the first author extended the concept to nonconvex closed sets. It was proven in [1] that the $V$-proximal normal cone is characterised in terms of the generalised projection as follows:

$$
\begin{aligned}
x^{*} \in N^{\pi}(S ; \bar{x}) & \Leftrightarrow \exists \rho>0, \text { such that } \bar{x} \in \pi_{S}\left(J(\bar{x})+\rho x^{*}\right) ; \\
& \Leftrightarrow \exists \sigma>0, \text { such that }\left\langle x^{*} ; x-\bar{x}\right\rangle \leq \sigma V(J(\bar{x}), x), \forall x \in S ; \\
& \Leftrightarrow \exists \delta, \sigma>0, \text { such that }\left\langle x^{*} ; x-\bar{x}\right\rangle \leq \sigma V(J(\bar{x}), x), \forall x \in S \cap(\bar{x}+\delta \mathbb{B}) .
\end{aligned}
$$

Here, $\mathbb{B}$ denotes the closed unit ball in $X$.

We also recall (see, for instance, [4]) the definition of the Fréchet subdifferential as follows: $x^{*} \in \partial^{F} f(\bar{x})$ if and only if for all $\epsilon>0$, there exists $\delta>0$ such that:

$$
\left\langle x^{*} ; x-\bar{x}\right\rangle \leq f(x)-f(\bar{x})+\epsilon\|x-\bar{x}\|, \quad \forall x \in \bar{x}+\delta \mathbb{B} .
$$

The Fréchet normal cone $N^{F}(S ; x)$ of a non-empty closed subset $S$ in $X$ at $\bar{x} \in S$ is defined as $N^{F}(S ; \bar{x})=\partial^{F} \psi_{S}(\bar{x})$.

\section{V-Proximal Trustworthy Spaces}

In [5], we introduced and studied the class of $V$-proximal trustworthy spaces as follows:

Definition 2 ([5]). We will say that a Banach space $X$ is $V$-proximal trustworthy provided that for any $\epsilon>0$, any two functions $f_{1}, f_{2}: X \rightarrow \mathbb{R} \cup\{\infty\}$, and any $u \in X$ such that $f_{1}$ is lower semicontinuous and $f_{2}$ is Lipschitz around $u$, the following fuzzy sum rule holds:

$$
\partial^{\pi}\left(f_{1}+f_{2}\right)(u) \subset \bigcup\left\{\partial^{\pi} f_{1}\left(u_{1}\right)+\partial^{\pi} f_{2}\left(u_{2}\right): u_{i} \in U_{f_{i}}(u, \epsilon), i=1,2\right\}+\epsilon \mathbb{B}_{*} .
$$

Here, $U_{f_{i}}(u, \epsilon):=\left\{x \in u+\epsilon \mathbb{B}\right.$ such that $\left.\left|f_{i}(x)-f_{i}(u)\right|<\epsilon\right\}$, and $\mathbb{B}_{*}$ denotes the closed unit ball in $X^{*}$.

We proved in [5] that all the integral spaces $L^{p}(1<p<\infty)$, as well as the sequence spaces $l^{p}(1<p<\infty)$, the Sobolev spaces $W^{p, n}(1<p<\infty)$, and the Schatten trace ideals $\mathbf{C}^{p}(1<p<\infty)$, are $V$-proximal trustworthy. The following results were proven in [6] for a particular class of a $V$-proximal trustworthy space, but their proofs are still valid for any $V$-proximal trustworthy space. We state them here without proofs.

Proposition 1 ([6]). Let X be a q-uniformly convex and p-uniformly smooth Banach space. Let $S$ be a closed subset of $X$ with $x \in S$, and let $x^{*} \in N^{F}(S ; x)$. Assume that $X$ is a $V$-proximal trustworthy space. Then, for any $\epsilon>0$, there exists $x_{\epsilon} \in(\bar{x}+\epsilon \mathbb{B}) \cap S$ such that $x^{*} \in N^{\pi}\left(S ; x_{\epsilon}\right)+\epsilon \mathbb{B}_{*}$.

Proposition 2 ([6]). Let $X$ be a $V$-proximal trustworthy space, and let $f: X \rightarrow \mathbb{R} \cup\{\infty\}$ be a proper l.s.c. function around $\bar{x} \in \operatorname{dom} f$. Then, for any $x^{*} \in X^{*}$ with $\left(x^{*} ; 0\right) \in N^{\pi}($ epif $;(\bar{x}, f(\bar{x})))$, there exist sequences $x_{k} \rightarrow \bar{x}$ with $f\left(x_{k}\right) \rightarrow f(\bar{x}), \lambda_{k} \rightarrow 0^{+}$, and $x_{k}^{*} \in \lambda_{k} \partial^{\pi} f\left(x_{k}\right)$ such that $\left\|x_{k}^{*}-x^{*}\right\|_{*} \rightarrow 0$.

\section{On Generalised V-Prox-Regular Sets}

We recall from [7] the following definition of the generalised $V$-prox-regularity for sets.

Definition 3 ([7]). Let $S$ be a non-empty closed set in a reflexive Banach space $X$, and let $\bar{x} \in S$. We will say that $S$ is generalised $V$-prox-regular at $\bar{x}$ if and only if there exist $r>0$ and $\epsilon>0$ such that for all 
$x \in S \cap(\bar{x}+\epsilon \mathbb{B})$ and for any $x^{*} \in N^{\pi}(S ; x) \cap \epsilon \mathbb{B}_{*}$, the point $x$ is a generalised projection of $J x+r x^{*}$ on $S \cap(\bar{x}+\epsilon \mathbb{B})$, that is $x \in \pi_{S \cap(\bar{x}+\epsilon \mathbb{B})}\left(J x+r x^{*}\right)$.

We start with the following characterisation of generalised prox-regular sets.

Theorem 1. Let $S$ be a closed subset in a reflexive smooth Banach space $X$, and let $\bar{x} \in S$. The two following assertions are equivalent:

1. $S$ is generalised $V$-prox-regular at $\bar{x}$;

2. There exist $r>0$ and $\epsilon>0$ such that for all $x \in S \cap(\bar{x}+\epsilon \mathbb{B})$ and all $x^{*} \in N^{\pi}(S ; x) \cap \epsilon \mathbb{B}$, we have:

$$
\left\langle x^{*} ; y-x\right\rangle \leq \frac{4(\|\bar{x}\|+\epsilon)+r}{2 r} d_{S}(y)+\frac{1}{2 r} V(J x ; y), \quad \forall y \in \bar{x}+\frac{\epsilon}{2} \mathbb{B} .
$$

Proof. $(1) \Rightarrow(2)$. Assume that $S$ is generalised $V$-prox-regular at $\bar{x}$. Then, there exist some $\epsilon \in(0,1)$ and $r>0$ such that $\forall x \in(\bar{x}+\epsilon \mathbb{B}) \cap S$ and any $x^{*} \in N^{\pi}(S ; x) \cap \epsilon \mathbb{B}$, we have $x \in \pi_{S \cap(\bar{x}+\epsilon \mathbb{B})}\left(J x+r x^{*}\right)$, that is,

$$
V\left(J x+r x^{*} ; x\right) \leq V\left(J x+r x^{*} ; y\right), \quad \forall y \in(\bar{x}+\epsilon \mathbb{B}) \cap S .
$$

We can easily check that the function $y \mapsto V\left(J x+r x^{*} ; y\right)$ is Lipschitz with ratio $K:=4(\|\bar{x}\|+\epsilon)+r$ on $(\bar{x}+\epsilon \mathbb{B}) \cap S$. Then, the Clarke penalisation ensures:

$$
V\left(J x+r x^{*} ; x\right) \leq V\left(J x+r x^{*} ; y\right)+K d_{(\bar{x}+\epsilon \mathbb{B}) \cap S}(y), \text { for all } y \in X .
$$

Observe that:

$$
\begin{aligned}
V\left(J x+r x^{*} ; x\right)-V\left(J x+r x^{*} ; y\right) & =\|x\|^{2}-\|y\|^{2}-2\left\langle J x+r x^{*} ; x-y\right\rangle \\
& =-\|y\|^{2}-\|x\|^{2}+2\langle J x ; y\rangle-2 r\left\langle x^{*} ; x-y\right\rangle \\
& =-V(J x ; y)-2 r\left\langle x^{*} ; x-y\right\rangle .
\end{aligned}
$$

Thus, the inequality (2) becomes:

$$
-V(J x ; y)-2 r\left\langle x^{*} ; x-y\right\rangle \leq K d_{(\bar{x}+\epsilon \mathbb{B}) \cap S}(y), \text { for all } y \in X .
$$

On the other hand, we can easily prove the following equality:

$$
d_{(\bar{x}+\epsilon \mathbb{B}) \cap S}(y)=d_{S}(y), \text { for all } y \in \bar{x}+\frac{\epsilon}{2} \mathbb{B} .
$$

Thus, we obtain:

$$
\left\langle x^{*} ; y-x\right\rangle \leq \frac{K}{2 r} d_{S}(y)+\frac{1}{2 r} V(J x ; y), \quad \forall y \in \bar{x}+\frac{\epsilon}{2} \mathbb{B},
$$

and hence, the proof of $(1) \Rightarrow(2)$ is complete.

$(2) \Rightarrow(1)$. Assume now that there exist $r>0$ and $\epsilon_{1}>0$ such that (2) holds. Then, for all $x \in\left(\bar{x}+\epsilon_{1} \mathbb{B}\right) \cap S$ and all $x^{*} \in N^{\pi}(S ; x) \cap \epsilon_{1} \mathbb{B}$, we have:

$$
\left\langle x^{*} ; y-x\right\rangle \leq \frac{4\left(\|\bar{x}\|+\epsilon_{1}\right)+r}{2 r} d_{S}(y)+\frac{1}{2 r} V(J x ; y), \quad \forall y \in \bar{x}+\frac{\epsilon_{1}}{2} \mathbb{B} .
$$

Then:

$$
\left\langle x^{*} ; y-x\right\rangle \leq \frac{1}{2 r} V(J x ; y), \quad \forall y \in\left(\bar{x}+\frac{\epsilon_{1}}{2} \mathbb{B}\right) \cap S .
$$


Using (3), we obtain:

$$
V\left(J x+r x^{*} ; x\right) \leq V\left(J x+r x^{*} ; y\right), \quad \forall y \in\left(\bar{x}+\frac{\epsilon_{1}}{2} \mathbb{B}\right) \cap S,
$$

that is, $x \in \pi_{S \cap\left(\bar{x}+\frac{\epsilon_{1}}{2} \mathbb{B}\right)}\left(J x+r x^{*}\right)$. Set $\epsilon:=\frac{\epsilon_{1}}{2}$. Therefore, for all $x \in S \cap(\bar{x}+\epsilon \mathbb{B})$ and all $x^{*} \epsilon$ $N^{\pi}(S ; x) \cap \epsilon \mathbb{B}$, we have $x \in \pi_{S \cap(\bar{x}+\epsilon \mathbb{B})}\left(J x+r x^{*}\right)$. This means by definition that $S$ is generalised $V$-prox-regular at $\bar{x}$, and hence, the proof is finished.

We present the first consequence of the previous theorem, in which we prove the closedness of the $V$-proximal normal cone, as a set-valued mapping, for the class of generalised $V$-prox-regular sets. We point out that this kind of property is very important in applications such as nonconvex sweeping processes and variational problems (see [4] in the Hilbert space setting).

Theorem 2. Let $S$ be a closed subset in a reflexive smooth Banach space, and let $\bar{x} \in S$. Assume that $S$ is generalised V-prox-regular at $\bar{x}$. Then, $N^{\pi}(S ; \bar{x})=N^{L \pi}(S ; \bar{x})$.

Proof. Since, obviously, the inclusion $N^{\pi}(S ; \bar{x}) \subset N^{L \pi}(S ; \bar{x})$ is always true, we have to prove the reverse inclusion $N^{L \pi}(S ; \bar{x}) \subset N^{\pi}(S ; \bar{x})$. Assume that $S$ is generalised $V$-prox-regular at $\bar{x}$. Then, by Theorem 1, there exist $r>0$ and $\epsilon>0$ such that for all $x \in S \cap(\bar{x}+\epsilon \mathbb{B})$ and any $x^{*} \in N^{\pi}(S ; x) \cap \epsilon \mathbb{B}$, we have:

$$
\left\langle x^{*} ; y-x\right\rangle \leq \frac{4(\|\bar{x}\|+\epsilon)+r}{2 r} d_{S}(y)+\frac{1}{2 r} V(J x ; y), \quad \forall y \in \bar{x}+\frac{\epsilon}{2} \mathbb{B} .
$$

Fix now any $z^{*} \in N^{L \pi}(S ; \bar{x})$. There exist $x_{n} \rightarrow \bar{x}$ with $x_{n} \in S$, and $z_{n}^{*}$ weakly converges to $z^{*}$ with $z_{n}^{*} \in N^{\pi}\left(S ; x_{n}\right)$. For $n$ sufficiently large, we have $x_{n} \in S \cap(\bar{x}+\epsilon \mathbb{B})$, and so, for any $y \in \bar{x}+\frac{\epsilon}{2} \mathbb{B}$, the above inequality ensures:

$$
\left\langle\frac{\epsilon z_{n}^{*}}{\left\|z_{n}^{*}\right\|+1} ; y-x_{n}\right\rangle \leq \frac{4(\|\bar{x}\|+\epsilon)+r}{2 r} d_{S}(y)+\frac{1}{2 r} V\left(J x_{n} ; y\right) .
$$

This ensures that:

$$
\left\langle z_{n}^{*} ; y-x_{n}\right\rangle \leq \frac{(4(\|\bar{x}\|+\epsilon)+r)\left(\left\|z_{n}^{*}\right\|+1\right)}{2 r \epsilon} d_{S}(y)+\frac{\left(\left\|z_{n}^{*}\right\|+1\right)}{2 \epsilon r} V\left(J x_{n} ; y\right), \quad \forall y \in \bar{x}+\frac{\epsilon}{2} \mathbb{B} .
$$

Thus, for any $y \in S \cap\left(\bar{x}+\frac{\epsilon}{2} \mathbb{B}\right)$, we have:

$$
\left\langle z_{n}^{*} ; y-x_{n}\right\rangle \leq \frac{\left(\left\|z_{n}^{*}\right\|+1\right)}{2 \epsilon r} V\left(J x_{n} ; y\right) .
$$

The weak convergence of $z_{n}^{*}$ to $z^{*}$ ensures its boundedness, that is, for some $L>0$, we have $\left\|z_{n}^{*}\right\| \leq L, \forall n$. Therefore,

$$
\left\langle z_{n}^{*} ; y-x_{n}\right\rangle \leq \frac{(L+1)}{2 \epsilon r} V\left(J x_{n} ; y\right), \forall y \in S \cap\left(\bar{x}+\frac{\epsilon}{2} \mathbb{B}\right) .
$$

Now, by passing to the limit in the last inequality when $n \rightarrow \infty$, and by taking into account the fact that $V$ and $J$ are continuous, we obtain:

$$
\left\langle z^{*} ; y-\bar{x}\right\rangle \leq \frac{(L+1)}{2 \epsilon r} V(J \bar{x} ; y), \forall y \in S \cap\left(\bar{x}+\frac{\epsilon}{2} \mathbb{B}\right) .
$$

This ensures by (1) that $z^{*} \in N^{\pi}(S ; \bar{x})$, and hence, the proof is complete.

Now, we recall the definition of uniformly generalised $V$-prox-regular sets, which is the uniform concept of the one given in Definition 3. 
Definition 4 ([7]). Let $S$ be a non-empty closed set in a reflexive Banach space $X$. We will say that $S$ is uniformly generalised $V$-prox-regular if and only if there exist $r>0$ such that for all $x \in S$ and for any $x^{*} \in N^{\pi}(S ; x)$ with $\left\|x^{*}\right\|<1$, the point $x$ is a generalised projection of $J x+r x^{*}$ on $S$, that is $x \in \pi_{S}\left(J x+r x^{*}\right)$.

Obviously, if $S$ is a uniformly generalised $V$-prox-regular set, then it is generalised $V$-prox-regular at any point $x \in S$, and hence, by Theorem 2 , we have for any $x \in S$ the equality $N^{\pi}(S ; x)=N^{L \pi}(S ; x)$. We prove an analogous characterisation to the one proven in Theorem 1 for uniformly generalised $V$-prox-regular sets.

Theorem 3. Let $S$ be a closed non-empty subset in a reflexive smooth Banach space X. Then, the following assertions are equivalent:

1. $S$ is uniformly generalised $V$-prox-regular;

2. There exist $r>0$ and $\alpha_{0}>0$ such that for all $\alpha \geq \alpha_{0}$, for all $x \in S$, and any $x^{*} \in N^{\pi}(S ; x)$ with $\left\|x^{*}\right\|<1$, we have:

$$
\left\langle x^{*} ; y-x\right\rangle \leq \frac{\|x\|+r+3 \alpha}{r} d_{S}(y)+\frac{1}{2 r} V(J x ; y), \quad \forall y \in \alpha \mathbb{B} .
$$

3. There exist $r>0$ and $\alpha_{0}>0$ such that for all $\alpha \geq \alpha_{0}$, for all $x \in S$, and any $x^{*} \in N^{L \pi}(S ; x)$ with $\left\|x^{*}\right\|<1$, we have that (4) holds.

Proof. We start with the implication $(1) \Rightarrow(2)$. Assume that $S$ is uniformly generalised $V$-prox-regular. Let $r>0$ be given as in Definition 4. Fix any $x \in S$ and any $x^{*} \in N^{\pi}(S ; x)$ with $\left\|x^{*}\right\|<1$ such that:

$$
V\left(J x+r x^{*} ; x\right) \leq V\left(J x+r x^{*} ; s\right), \text { for all } s \in S .
$$

Then, by using (3), we obtain:

$$
\left\langle x^{*} ; s-x\right\rangle \leq \frac{1}{2 r} V(J x ; s), \text { for all } s \in S .
$$

Let $\alpha_{0}>0$ such that $S \cap \alpha_{0} \mathbb{B} \neq \varnothing\left(\alpha_{0}\right.$ exists since $\left.S \neq \varnothing\right)$. Let $\alpha \geq \alpha_{0}$ so that $S \cap \alpha \mathbb{B} \neq \varnothing$. Let $h(s):=$ $\left\langle x^{*} ; s-x\right\rangle-\frac{1}{2 r} V(J x ; s)$, for all $s \in X$. We can show easily that $h$ is Lipschitz on $S \cap 3 \alpha \mathbb{B}$ with ratio $K:=\frac{r+3 \alpha+\|x\|}{r}$. Therefore, using Clarke penalisation, the inequality (5) becomes:

$$
\left\langle x^{*} ; y-x\right\rangle \leq \frac{1}{2 r} V(J x ; y)+K d_{S \cap 3 \alpha \mathbb{B}}(y), \text { for all } y \in X .
$$

Since we can easily show that $d_{S \cap 3 \alpha \mathbb{B}}(y)=d_{S}(y)$, for all $y \in \alpha \mathbb{B}$, we obtain:

$$
\left\langle x^{*} ; y-x\right\rangle \leq \frac{1}{2 r} V(J x ; y)+K d_{S}(y), \text { for all } y \in \alpha \mathbb{B},
$$

and hence, the proof of $(1) \Rightarrow(2)$ is complete. We prove the reverse implication $(2) \Rightarrow(1)$. Assume that there exist $r>0, \alpha_{0}>0$ with $S \cap \alpha_{0} \mathbb{B} \neq \varnothing$ such that $\forall \alpha \geq \alpha_{0}, \forall x \in S, \forall x^{*} \in N^{\pi}(S ; x)$ with $\left\|x^{*}\right\|<1$, we have:

$$
\left\langle x^{*} ; y-x\right\rangle \leq \frac{1}{2 r} V(J x ; y)+\frac{r+3 \alpha+\|x\|}{r} d_{S}(y), \text { for all } y \in \alpha \mathbb{B} .
$$

Then:

$$
\left\langle x^{*} ; y-x\right\rangle \leq \frac{1}{2 r} V(J x ; y), \text { for all } y \in \alpha \mathbb{B} \cap S,
$$

and so, by (3), once again, we obtain:

$$
V\left(J x+r x^{*} ; x\right) \leq V\left(J x+r x^{*} ; y\right), \text { for all } y \in \alpha \mathbb{B} \cap S .
$$


Let $y^{*}:=J x+r x^{*}$ and $M:=\max \left\{\|x\|+r ; \alpha_{0}\right\}$. Clearly, we have $\left\|y^{*}\right\| \leq M$ and $S \cap M \mathbb{B} \neq \varnothing$ (since $M \geq \alpha_{0}$ and $S \cap \alpha_{0} \mathbb{B} \neq \varnothing$ ). Therefore, by Lemma 2.12 in [7], we obtain:

$$
\inf _{y \in S} V\left(y^{*} ; y\right)=\inf _{y \in S \cap 3 M \mathbb{B}} V\left(y^{*} ; y\right) .
$$

Take $x \in S \cap 3 M \mathbb{B}$ and $\alpha=3 M$ in (6); we get:

$$
V\left(J x+r x^{*} ; x\right)=\inf _{y \in S \cap \alpha \mathbb{B}} V\left(J x+r x^{*} ; y\right)=\inf _{y \in S} V\left(J x+r x^{*} ; y\right) .
$$

This means that $x \in \pi_{S}\left(J x+r x^{*}\right)$. Since this relation holds $\forall x \in S, \forall x^{*} \in N^{\pi}(S ; x)$ with $\left\|x^{*}\right\|<1$, we deduce that $S$ is uniformly generalised $V$-prox-regular with ratio $r>0$. The proof of $(2) \Longleftrightarrow(3)$ follows from the fact that $N^{\pi}(S ; x)=N^{L \pi}(S ; x)$, proven in Theorem 2 .

The case of bounded sets is treated in the following corollary, and its proof is simpler than the proof of Theorem 3.

Corollary 1. Let $S$ be a closed bounded subset in a reflexive smooth Banach space X with $S \subset M \mathbb{B}$. Then, the following assertions are equivalent:

1. $S$ is uniformly generalised $V$-prox-regular;

2. There exists $r>0$ such that for any $x \in S$ and any $x^{*} \in N^{\pi}(S ; x)$ with $\left\|x^{*}\right\|<1$, we have:

$$
\left\langle x^{*} ; y-x\right\rangle \leq \frac{2 M+r}{r} d_{S}(y)+\frac{1}{2 r} V(J x ; y), \quad \forall y \in X .
$$

3. There exists $r>0$ such that for any $x \in S$ and any $x^{*} \in N^{\pi}(S ; x)$ with $\left\|x^{*}\right\|<1$, we have that (7) holds.

Proof. As in the proof of Theorem 3, the equivalence (2) $\Longleftrightarrow(3)$ follows from Theorem 2. Therefore, we have only to prove that $(1) \Longleftrightarrow(2)$. Assume that $S$ is uniformly generalised $V$-prox-regular. Let $r>0$ be given as in Definition 4. Fix any $x \in S$ and any $x^{*} \in N^{\pi}(S ; x)$ with $\left\|x^{*}\right\|<1$ such that:

$$
V\left(J x+r x^{*} ; x\right) \leq V\left(J x+r x^{*} ; s\right), \text { for all } s \in S .
$$

Then, by (3), we obtain:

$$
\left\langle x^{*} ; s-x\right\rangle \leq \frac{1}{2 r} V(J x ; s), \text { for all } s \in S .
$$

Let $h(s):=\left\langle x^{*} ; s-x\right\rangle-\frac{1}{2 r} V(J x ; s)$, for all $s \in X$. Clearly, $h$ is Lipschitz on $S$ with ratio $K:=\frac{2 M+r}{r}$. Therefore, using Clarke penalisation, the inequality (8) becomes:

$$
\left\langle x^{*} ; y-x\right\rangle \leq \frac{1}{2 r} V(J x ; y)+K d_{S}(y), \text { for all } y \in X,
$$

and hence, the proof of $(1) \Rightarrow(2)$ is achieved. We prove the reverse implication (2) $\Rightarrow(1)$. Assume that (7) holds. Then, there exists $r>0$ such that $\forall x \in S, \forall x^{*} \in N^{\pi}(S ; x)$ with $\left\|x^{*}\right\|<1$, we have:

$$
\left\langle x^{*} ; y-x\right\rangle \leq \frac{1}{2 r} V(J x ; y)+\frac{2 M+r}{r} d_{S}(y), \text { for all } y \in X .
$$

Then:

$$
\left\langle x^{*} ; y-x\right\rangle \leq \frac{1}{2 r} V(J x ; y), \text { for all } y \in S,
$$

and so, by (3), we obtain:

$$
V\left(J x+r x^{*} ; x\right) \leq V\left(J x+r x^{*} ; y\right), \text { for all } y \in S .
$$


This means $x \in \pi_{S}\left(J x+r x^{*}\right), \forall x \in S, \forall x^{*} \in N^{\pi}(S ; x)$ with $\left\|x^{*}\right\|<1$. Thus, $S$ is uniformly generalised $V$-prox-regular with ratio $r>0$, and hence, the proof is complete.

\section{4. $V$-Primal Lower Nice Functions}

In this section, we start by adapting the definition of primal lower nice functions given in [8]. We use the functional $V$ instead of the norm square and the geometric $V$-prox-subdifferential $\partial_{G}^{\pi}$ instead of the usual proximal subdifferential.

Definition 5. Let $f: X \rightarrow \mathbb{R} \cup\{\infty\}$ be an l.s.c. function. We will say that $f$ is $V$-primal lower nice (V-p.l.n.) at $\bar{x} \in \operatorname{domf}$, if there exist $\lambda_{1}, \lambda_{2}>0, c>0, T>0$ such that:

$$
f(y) \geq f(x)+\left\langle x^{*} ; y-x\right\rangle-\frac{t}{2} V(J x, y)
$$

whenever $t \geq T, x \in \mathcal{N}_{V}\left(\bar{x}, \lambda_{1}, \lambda_{2}\right), y \in \bar{x}+\lambda_{1} \mathbb{B}, x^{*} \in \partial_{G}^{\pi} f(x)$, and $\left\|x^{*}\right\| \leq$ ct. Here:

$$
\mathcal{N}_{V}\left(\bar{x}, \lambda_{1}, \lambda_{2}\right):=\left\{x \in \bar{x}+\lambda_{1} \mathbb{B} \text { with } V(J \bar{x}, x) \leq \lambda_{2}^{2}\right\}
$$

We notice that this definition extends, to reflexive smooth Banach spaces, the definition of primal lower nice functions defined in Hilbert spaces in [8] and in finite dimension spaces in [9]. In the following proposition, we start by proving an important property of the class of $V$-primal lower nice functions, which is the $J$-hypomonotonicity of the geometric $V$-prox-subdifferential $\partial_{G}^{\pi}$ in the sense of [7]. We point out that a different extension to Banach spaces was given in [8] and in [10] (see also [11,12]).

Proposition 3. Assume that $X$ is a reflexive smooth Banach space, and let $f: X \rightarrow \mathbb{R} \cup\{\infty\}$ be an l.s.c. function. For any $V$-p.l.n. function $f$ at $\bar{x} \in \operatorname{dom} f$, there exist $\lambda>0, c>0, T>0$ such that the set-valued mapping $x \mapsto \partial_{G}^{\pi} f(x) \cap c t \mathbb{B}$ is J-hypomonotone with any ratio $t \geq T$ over $\bar{x}+\lambda \mathbb{B}$, that is for any $t \geq T$, any $x_{1}, x_{2} \in \bar{x}+\lambda \mathbb{B}, x_{i}^{*} \in \partial_{G}^{\pi} f\left(x_{i}\right), i=1,2$, with $\max \left\{\left\|x_{1}^{*}\right\|,\left\|x_{2}^{*}\right\|\right\} \leq c t$, we have:

$$
\left\langle x_{1}^{*}-x_{2}^{*} ; x_{1}-x_{2}\right\rangle \geq-t\left\langle J x_{1}-J x_{2} ; x_{1}-x_{2}\right\rangle .
$$

Proof. Observe that for any $\epsilon_{1}, \epsilon_{2}>0$, there exists $\epsilon>0$ such that:

$$
\bar{x}+\epsilon \mathbb{B} \subset \mathcal{N}_{V}\left(\bar{x}, \epsilon_{1}, \epsilon_{2}\right) .
$$

Assume that $f$ is $V$-p.l.n. at $\bar{x} \in \operatorname{dom} f$. Then, there are $\lambda>0, c>0, T>0$ such that (9) holds for any $t \geq T, x_{1}, x_{2} \in \bar{x}+\lambda \mathbb{B}, x_{i}^{*} \in \partial_{G}^{\pi} f\left(x_{i}\right), i=1,2$, and $\max \left\{\left\|x_{1}^{*}\right\|,\left\|x_{2}^{*}\right\|\right\} \leq c t$. Thus:

$$
f\left(x_{1}\right) \geq f\left(x_{2}\right)+\left\langle x_{2}^{*} ; x_{1}-x_{2}\right\rangle-\frac{t}{2} V\left(J x_{2}, x_{1}\right)
$$

and:

$$
f\left(x_{2}\right) \geq f\left(x_{1}\right)+\left\langle x_{1}^{*} ; x_{2}-x_{1}\right\rangle-\frac{t}{2} V\left(J x_{1}, x_{2}\right) .
$$

Adding these two inequalities gives:

$$
0 \geq\left\langle x_{1}^{*}-x_{2}^{*} ; x_{2}-x_{1}\right\rangle-\frac{t}{2} V\left(J x_{1}, x_{2}\right)-\frac{t}{2} V\left(J x_{2}, x_{1}\right) .
$$

On the other hand, we have:

$$
\begin{aligned}
V\left(J x_{1}, x_{2}\right)+V\left(J x_{2}, x_{1}\right) & =2\left\|x_{1}\right\|^{2}-2\left[\left\langle J x_{1} ; x_{2}\right\rangle+\left\langle J x_{2} ; x_{1}\right\rangle\right]+2\left\|x_{2}\right\|^{2} \\
& =2\left[\left\langle J x_{1} ; x_{1}\right\rangle-\left\langle J x_{1} ; x_{2}\right\rangle-\left\langle J x_{2} ; x_{1}\right\rangle+\left\langle J x_{2} ; x_{2}\right\rangle\right]
\end{aligned}
$$




$$
=2\left\langle J x_{1}-J x_{2} ; x_{1}-x_{2}\right\rangle \text {. }
$$

Therefore, (11) becomes:

$$
\left\langle x_{1}^{*}-x_{2}^{*} ; x_{1}-x_{2}\right\rangle \geq-t\left\langle J x_{1}-J x_{2} ; x_{1}-x_{2}\right\rangle
$$

and hence, the proof is complete.

In the next theorem, we prove that the reverse implication of the previous proposition also holds in $q$-uniformly convex and $p$-uniformly smooth Banach spaces, which is $V$-proximal trustworthy. We state the following proposition from [7], which is needed in our next proof.

Proposition 4 ([7]). If $X$ is $q$-uniformly convex and p-uniformly smooth, then $\forall \alpha>0, \exists K_{1}, K_{2}, K_{3}>0$ such that for all $x, y \in \alpha \mathbb{B}$ :

$$
\langle J x-J y ; x-y\rangle \geq K_{1}\|x-y\|^{q},\|J x-J y\| \leq K_{2}\|x-y\|^{p-1}, \text { and } V(J x, y) \geq K_{3}\|x-y\|^{q} .
$$

In the proof of the following theorem, we use techniques and ideas from [10].

Theorem 4. Assume that $X$ is $q$-uniformly convex and p-uniformly smooth, admitting which is $V$-proximal trustworthy. The function $f$ is V-p.l.n. at $x_{0} \in \operatorname{dom} f$ if and only if (10) holds.

Proof. We prove only the reverse implication. First, we prove the following claim. Assume that there are $\lambda>0, c>0, T>0$ such that (10) holds. Set $M:=\|\bar{x}\|+4 \lambda$. Then, by Proposition 4 , there exist $K_{1}, K_{2}, K_{3}>0$ such that for all $x, y \in M \mathbb{B}$ :

$$
\begin{aligned}
\langle J x-J y ; x-y\rangle & \geq K_{1}\|x-y\|^{q} \\
\|J x-J y\| & \leq K_{2}\|x-y\|^{p-1} \\
V(J x, y) & \geq K_{3}\|x-y\|^{q} .
\end{aligned}
$$

The constants $K_{1}, K_{2}$, and $K_{3}$ depend only on the space $X$ and the constant $M>0$.

Claim 1. Take $\lambda^{\prime} \in\left(0, \min \left\{\frac{c}{2}, \frac{1}{4}\left(\frac{c}{16 K_{2}}\right)^{\frac{1}{p-1}}, \frac{\lambda}{4}\right\}\right)$. Take $\lambda_{2} \in\left(0, \sqrt{2^{q-1} \lambda^{\prime q} K_{3}}\right)$. Fix $c^{\prime}$ and $T^{\prime}$ in a such way that:

$$
0<c^{\prime}<\min \left\{\frac{K_{3}\left(2 \lambda^{\prime}\right)^{q-1}}{32}, \frac{\lambda^{\prime}}{8}\right\}
$$

and:

$$
T^{\prime}>\max \left\{2 T, \frac{2[1+f(\bar{x})-K]}{K_{3} \lambda^{\prime q}}\right\} \text {. }
$$

We claim that for arbitrary $x_{0} \in \mathcal{N}_{V}\left(\bar{x}, \lambda^{\prime}, \lambda_{2}\right)$ and $x^{*} \in \partial_{G}^{\pi} f\left(x_{0}\right)$ such that $\left\|x^{*}\right\| \leq c^{\prime} t$ and $t \geq T^{\prime}$ and arbitrary $y_{0} \in \bar{x}+4 \lambda^{\prime} \mathbb{B}$ such that:

$$
f\left(y_{0}\right)-1+\left\langle x^{*} ; x_{0}-y_{0}\right\rangle+\frac{t}{2} V\left(J x_{0}, y_{0}\right)<\inf _{y \in \bar{x}+4 \lambda^{\prime} \mathbb{B}}\left\{f(y)+\left\langle x^{*} ; x_{0}-y\right\rangle+\frac{t}{2} V\left(J x_{0}, y\right)\right\},
$$

it follows that $y_{0} \in \bar{x}+3 \lambda^{\prime} \mathbb{B}$.

Proof of Claim 1. Assume the contrary, that is there are some $x^{\prime} \in \mathcal{N}_{V}\left(\bar{x}, \lambda^{\prime}, \lambda_{2}\right), v^{*} \in \partial_{G}^{\pi} f\left(x^{\prime}\right)$ with $\left\|v^{*}\right\| \leq c^{\prime} t$, for some $t \geq T^{\prime}$ and $y^{\prime} \in\left[\bar{x}+4 \lambda^{\prime} \mathbb{B}\right] \backslash\left[\bar{x}+3 \lambda^{\prime} \mathbb{B}\right]$ such that:

$$
f\left(y^{\prime}\right)+\left\langle v^{*} ; x^{\prime}-y^{\prime}\right\rangle+\frac{t}{2} V\left(J x^{\prime}, y^{\prime}\right)<f(\bar{x})+\left\langle v^{*} ; x^{\prime}-\bar{x}\right\rangle+\frac{t}{2} V\left(J x^{\prime}, \bar{x}\right)+1 .
$$


Therefore,

$$
1+f(\bar{x})-K>\left\langle v^{*} ; \bar{x}-y^{\prime}\right\rangle+\frac{t}{2}\left[V\left(J x^{\prime}, y^{\prime}\right)-V\left(J x^{\prime}, \bar{x}\right)\right] .
$$

Since $x^{\prime} \in \bar{x}+\lambda^{\prime} \mathbb{B}$ and $y^{\prime} \in \bar{x}+4 \lambda^{\prime} \mathbb{B}$, we have $\left\|y^{\prime}\right\| \leq\|\bar{x}\|+4 \lambda^{\prime}<M$ and $\left\|x^{\prime}\right\|<M$, and hence, we can write by (14):

$$
V\left(J x^{\prime}, y^{\prime}\right) \geq K_{3}\left\|x^{\prime}-y^{\prime}\right\|^{q} \geq K_{3}\left(\left\|y^{\prime}-\bar{x}\right\|-\left\|x^{\prime}-\bar{x}\right\|\right)^{q} \geq K_{3}\left(3 \lambda^{\prime}-\lambda^{\prime}\right)^{q}=K_{3} 2^{q} \lambda^{\prime q}
$$

Therefore:

$$
V\left(J x^{\prime}, y^{\prime}\right)-V\left(J x^{\prime}, \bar{x}\right) \geq K_{3} 2^{q} \lambda^{\prime q}-\lambda_{2}^{2} \geq \frac{1}{2} K_{3}\left(2 \lambda^{\prime}\right)^{q} .
$$

Furthermore, we have:

$$
\left\langle v^{*} ; \bar{x}-y^{\prime}\right\rangle \geq-\left\|v^{*}\right\| \cdot\left\|y^{\prime}-\bar{x}\right\| \geq-4 \lambda^{\prime}\left\|v^{*}\right\| \geq-4 \lambda^{\prime} c^{\prime} t
$$

Thus:

$$
1+f(\bar{x})-K>-4 \lambda^{\prime} c^{\prime} t+\frac{t}{2}\left[\frac{1}{2} K_{3}\left(2 \lambda^{\prime}\right)^{q}\right]=t \lambda^{\prime}\left[-4 c^{\prime}+\frac{(2 \lambda)^{q-1} K_{3}}{2}\right] .
$$

From the choice of $c^{\prime}$ in (15), we have:

$$
-4 c^{\prime}>-\frac{K_{3}\left(2 \lambda^{\prime}\right)^{q-1}}{8}
$$

Hence, by (16), we obtain:

$$
\begin{aligned}
1+f(\bar{x})-K & >t \lambda^{\prime}\left[\frac{\left(2 \lambda^{\prime}\right)^{q-1} K_{3}}{4}\right]>T^{\prime} \lambda^{\prime}\left[\frac{\left(2 \lambda^{\prime}\right)^{q-1} K_{3}}{4}\right] \\
& >\lambda^{\prime}\left[\frac{\left(2 \lambda^{\prime}\right)^{q-1} K_{3}}{4}\right] \frac{2[1+f(\bar{x})-K]}{K_{3} \lambda^{\prime q}}>1+f(\bar{x})-K .
\end{aligned}
$$

This is a contradiction. Therefore, the proof of the claim is achieved.

Let us prove now the opposite direction of the theorem. Assume that (9) holds for some $\lambda>$ $0, c>0, T>0$. Let $\lambda^{\prime}, c^{\prime}, T^{\prime}$, and $\lambda_{2}$ be fixed as in Claim 1. Assume that the function $f$ is not $V$-p.l.n. at $\bar{x}$. Then, by definition, there are $x_{0} \in \mathcal{N}_{V}\left(\bar{x}, \lambda^{\prime}, \lambda_{2}\right), x^{*} \in \partial_{G}^{\pi} f\left(x_{0}\right)$ with $\left\|x^{*}\right\| \leq c^{\prime} t$ such that $t \geq T^{\prime}$ and some $y_{0} \in x_{0}+\lambda^{\prime} \mathbb{B}$ such that:

$$
f\left(y_{0}\right)<f\left(x_{0}\right)+\left\langle x^{*} ; y_{0}-x_{0}\right\rangle-\frac{t}{2} V\left(J x_{0}, y_{0}\right) .
$$

Therefore,

$$
\begin{aligned}
f\left(x_{0}\right) & >f\left(y_{0}\right)+\left\langle x^{*} ; x_{0}-y_{0}\right\rangle+\frac{t}{2} V\left(J x_{0}, y_{0}\right) \\
& >\inf _{y \in \bar{x}+4 \lambda^{\prime} \mathbb{B}}\left\{f(y)+\left\langle x^{*} ; x_{0}-y\right\rangle+\frac{t}{2} V\left(J x_{0}, y\right)\right\} .
\end{aligned}
$$

Define now the function $h$ on $X$ as:

$$
h(y):=f(y)+\left\langle x^{*} ; x_{0}-y\right\rangle+\frac{t}{2} V\left(J x_{0}, y\right)+\psi_{\left[\bar{x}+4 \lambda^{\prime} \mathbb{B}\right]}(y) .
$$

Clearly, the inequality (17) gives:

$$
f\left(x_{0}\right)>\inf _{y \in X} h(y)
$$


and so, by the definition of the infimum, we can find a sequence $y_{n} \in \bar{x}+4 \lambda^{\prime} \mathbb{B}$ such that:

$$
h\left(y_{n}\right)<\inf _{y \in X} h(y)+\frac{1}{n}
$$

Obviously, (17) ensures that $\left\|y_{n}-x_{0}\right\|$ does not converge to zero, that is there exists some positive number $\alpha>0$ such that $\left\|y_{n}-x_{0}\right\|>\alpha$, for $n$ sufficiently large. Clearly, $y_{n}$ satisfies Claim 1 , that is,

$$
\begin{aligned}
f\left(y_{n}\right)+\left\langle x^{*} ; x_{0}-y_{n}\right\rangle+\frac{t}{2} V\left(J x_{0}, y_{n}\right) & <\inf _{y \in \bar{x}+4 \lambda^{\prime} \mathbb{B}} h(y)+\frac{1}{n} \\
& <\inf _{y \in \bar{x}+4 \lambda^{\prime} \mathbb{B}} h(y)+1
\end{aligned}
$$

with $x_{0} \in \mathcal{N}_{V}\left(\bar{x}, \lambda^{\prime}, \lambda_{2}\right), x^{*} \in \partial_{G}^{\pi} f\left(x_{0}\right),\left\|x^{*}\right\|<c^{\prime} t, t \geq T^{\prime}, y_{n} \in \bar{x}+4 \lambda^{\prime} \mathbb{B}$.

Therefore, we have $y_{n} \in \bar{x}+3 \lambda^{\prime} \mathbb{B}$. Since $y_{n}$ satisfies the inequality (18), we can choose by the variational principle a sequence $x_{n} \in X$ with $\left\|x_{n}-y_{n}\right\| \leq \frac{1}{\sqrt{n}}$, and $x_{n}$ is the minimum of the function $h+\frac{1}{\sqrt{n}} \|$. $-x_{n} \|$. Therefore, $0 \in \partial^{\pi}\left[h+\frac{1}{\sqrt{n}}\left\|\cdot-x_{n}\right\|\right]\left(x_{n}\right)$. By the fuzzy sum rule for the $\partial^{\pi}$ in trustworthy spaces in Definition 2, we get two sequences $u_{n}, v_{n} \in x_{n}+\frac{1}{\sqrt{n}} \mathbb{B}$ with $\left|h\left(u_{n}\right)-h\left(x_{n}\right)\right|<\frac{1}{\sqrt{n}}$ such that:

$$
0 \in \partial^{\pi} h\left(u_{n}\right)+\frac{1}{\sqrt{n}} \partial^{\pi}\left[\left\|\cdot-x_{n}\right\|\right]\left(v_{n}\right)+\frac{1}{\sqrt{n}} \mathbb{B}_{* \prime}
$$

that is, there exist $p_{n}^{*} \in \partial^{\pi} h\left(u_{n}\right), q_{n}^{*} \in \frac{1}{\sqrt{n}} \partial^{\pi}\left[\left\|\cdot-x_{n}\right\|\right]\left(v_{n}\right)$ such that $\left\|p_{n}^{*}+q_{n}^{*}\right\|<\frac{1}{\sqrt{n}}$. Using now Proposition 3.16 in [1], we get some $x_{n}^{*} \in \partial^{\pi} f\left(u_{n}\right)$ such that $p_{n}^{*}=x_{n}^{*}-x^{*}+t\left[J u_{n}-J x_{0}\right]$ (i.e., $x_{n}^{*}=$ $\left.p_{n}^{*}+x^{*}-t\left[J u_{n}-J x_{0}\right]\right)$. Let $y_{n}^{*}:=q_{n}^{*}-x^{*}+t\left[J u_{n}-J x_{0}\right]$. Clearly, $\left|\left\|x_{n}^{*}\right\|-\left\|y_{n}^{*}\right\|\right| \rightarrow 0$. We need to estimate $\left\|x_{n}^{*}\right\|$. Observe that $\left\|q_{n}^{*}\right\| \leq \frac{1}{\sqrt{n}}$. We consider:

$$
\begin{aligned}
\left\|y_{n}^{*}\right\| & =\left\|x^{*}-q_{n}^{*}-t\left[J u_{n}-J x_{0}\right]\right\| \\
& \leq\left\|x^{*}\right\|+t\left\|J u_{n}-J x_{0}\right\|+\left\|q_{n}^{*}\right\| \\
& \leq c^{\prime} t+t\left\|J u_{n}-J x_{0}\right\|+\frac{1}{\sqrt{n}} .
\end{aligned}
$$

Observe that:

$$
\left\|u_{n}\right\| \leq\left\|u_{n}-x_{n}\right\|+\left\|x_{n}-y_{n}\right\|+\left\|y_{n}-\bar{x}\right\|+\|\bar{x}\| \leq \frac{2}{\sqrt{n}}+3 \lambda^{\prime}+\|\bar{x}\|<M,
$$

and so, both $x_{0}, u_{n} \in M \mathbb{B}$; so, by (13), we obtain:

$$
\left\|J u_{n}-J x_{0}\right\| \leq K_{2}\left\|u_{n}-x_{0}\right\|^{p-1} \leq K_{2}\left[\frac{2}{\sqrt{n}}+3 \lambda^{\prime}\right]^{p-1} \leq K_{2}\left[4 \lambda^{\prime}\right]^{p-1}
$$

for $n$ large enough. Thus:

$$
\left\|y_{n}^{*}\right\| \leq c^{\prime} t+t K_{2}\left[4 \lambda^{\prime}\right]^{p-1}+\frac{1}{\sqrt{n}}=t\left[c^{\prime}+K_{2}\left[4 \lambda^{\prime}\right]^{p-1}+\frac{1}{t \sqrt{n}}\right] .
$$

Clearly, for $n$ sufficiently large, we have $\frac{1}{t \sqrt{n}}<\frac{c}{16}$. Furthermore, we have $c^{\prime}<\frac{\lambda^{\prime}}{8}$ and $K_{2}\left[4 \lambda^{\prime}\right]^{p-1}<\frac{c}{16}$. Therefore:

$$
\left\|y_{n}^{*}\right\| \leq t\left[\frac{\lambda^{\prime}}{8}+\frac{c}{16}+\frac{c}{16}\right] \leq \frac{t c}{4}
$$


and hence:

$$
\left\|x_{n}^{*}\right\| \leq\left\|y_{n}^{*}\right\|+\left\|x_{n}^{*}+y_{n}^{*}\right\| \leq\left\|y_{n}^{*}\right\|+\left\|p_{n}^{*}+q_{n}^{*}\right\| \leq \frac{t c}{4}+\frac{1}{\sqrt{n}} \leq \frac{t c}{4}+\frac{t c}{8}<\frac{t c}{2},
$$

for $n$ large enough. Finally, we summarise: we obtained $x_{n}^{*} \in \partial^{\pi} f\left(u_{n}\right) \subset \partial_{G}^{\pi} f\left(u_{n}\right)$ with $\left\|x_{n}^{*}\right\|<\frac{c t}{2}$ and for $n$ sufficiently large:

$$
u_{n} \in x_{n}+\frac{1}{\sqrt{n}} \mathbb{B} \subset y_{n}+\frac{2}{\sqrt{n}} \mathbb{B} \subset \bar{x}+\left[3 \lambda^{\prime}+\frac{2}{\sqrt{n}}\right] \mathbb{B} \subset \bar{x}+\left[\frac{3}{4} \lambda+\frac{1}{\sqrt{n}}\right] \mathbb{B} \subset \bar{x}+\lambda \mathbb{B} .
$$

Furthermore, we have $x^{*} \in \partial_{G}^{\pi} f\left(x_{0}\right),\left\|x^{*}\right\| \leq c^{\prime} t<\frac{\lambda^{\prime}}{8} t<\frac{c}{16} t<c \frac{t}{2}, x_{0} \in \bar{x}+\lambda^{\prime} \mathbb{B} \subset \bar{x}+\lambda \mathbb{B}$, and $\frac{t}{2} \geq \frac{T^{\prime}}{2}>T$. Using (10), we obtain:

$$
\left\langle x_{n}^{*}-x^{*} ; u_{n}-x_{0}\right\rangle \geq-\frac{t}{2}\left\langle J u_{n}-J x_{0} ; u_{n}-x_{0}\right\rangle .
$$

We estimate the left-hand side:

$$
\begin{aligned}
\left\langle x_{n}^{*}-x^{*} ; u_{n}-x_{0}\right\rangle & =\left\langle x^{*}+y_{n}^{*} ; x_{0}-u_{n}\right\rangle-\left\langle y_{n}^{*}+x_{n}^{*} ; x_{0}-u_{n}\right\rangle \\
& \leq\left\langle x^{*}+y_{n}^{*} ; x_{0}-u_{n}\right\rangle+\left\|y_{n}^{*}+x_{n}^{*}\right\|\left\|x_{0}-u_{n}\right\| \\
& \leq\left\langle q_{n}^{*} ; x_{0}-u_{n}\right\rangle-t\left\langle J u_{n}-J x_{0} ; u_{n}-x_{0}\right\rangle+\left\|y_{n}^{*}+x_{n}^{*}\right\|\left\|x_{0}-u_{n}\right\| \\
& \leq \frac{2}{\sqrt{n}}\left\|x_{0}-u_{n}\right\|-t\left\langle J u_{n}-J x_{0} ; u_{n}-x_{0}\right\rangle
\end{aligned}
$$

Combining this inequality with (19), we obtain:

$$
-\frac{t}{2}\left\langle J u_{n}-J x_{0} ; u_{n}-x_{0}\right\rangle \leq-t\left\langle J u_{n}-J x_{0} ; u_{n}-x_{0}\right\rangle+\frac{2}{\sqrt{n}}\left\|x_{0}-u_{n}\right\|,
$$

and hence:

$$
\frac{t}{2}\left\langle J u_{n}-J x_{0} ; \frac{u_{n}-x_{0}}{\left\|x_{0}-u_{n}\right\|}\right\rangle \leq \frac{2}{\sqrt{n}} .
$$

Set $\alpha_{n}:=\left\langle J u_{n}-J x_{0} ; \frac{u_{n}-x_{0}}{\left\|x_{0}-u_{n}\right\|}\right\rangle$. The sequence $\left(\alpha_{n}\right)_{n}$ is a bounded positive sequence, so there exists some subsequence still labelled $\left(\alpha_{n}\right)_{n}$ such that $\lim _{n} \alpha_{n}=\bar{\alpha}$. Since $u_{n}, x_{0} \in M \mathbb{B}$, we have by (12):

$$
\alpha_{n} \geq K_{1}\left\|u_{n}-x_{0}\right\|^{q-1}
$$

If $\bar{\alpha}=0$, then $u_{n} \rightarrow x_{0}$, and so, $y_{n} \rightarrow x_{0}$, which is a contradiction with $\left\|y_{n}-x_{0}\right\|>\alpha>0$. Therefore, by taking the limit on both sides in (20), we get $\frac{t}{2} \leq 0$. This contradicts the fact that $t \geq T>0$, thus completing the proof of the theorem.

In the next theorem, we use Theorem 4 to prove that both $V$-prox-subdifferentials $\partial^{\pi} f$ and $\partial_{G}^{\pi} f$ coincide with their limit-subdifferentials for any $V$-primal lower nice function.

Theorem 5. Assume that X is q-uniformly convex and p-uniformly smooth, which is V-proximal trustworthy. For any $V$-p.l.n. function at $\bar{x}$, we have:

$$
\partial^{L P} f(\bar{x})=\partial^{L F} f(\bar{x})=\partial^{F} f(\bar{x})=\partial_{G}^{L \pi} f(\bar{x})=\partial^{L \pi} f(\bar{x})=\partial^{\pi} f(\bar{x})=\partial_{G}^{\pi} f(\bar{x}) .
$$

Here, $\partial^{L F} f(\bar{x})$ (resp. $\left.\partial^{L P} f(\bar{x})\right)$ denotes the limiting proximal (resp. Fréchet) subdifferential (for their definitions, we refer to [12]). 
Proof. The proof of the equalities $\partial^{L P} f(\bar{x})=\partial^{L F} f(\bar{x})=\partial^{L \pi} f(\bar{x})$ for any 1.s.c. functions was given in Theorem 5.1 in [6]. We assume that $f$ is $V$-p.l.n. at $\bar{x}$, and we prove the inclusion $\partial_{G}^{L \pi} f(\bar{x}) \subset \partial^{\pi} f(\bar{x})$. Let $x_{0}^{*} \in \partial_{G}^{L \pi} f(\bar{x})$. Then, there exist $x_{n} \rightarrow^{f} \bar{x}$ and $x_{n}^{*} \rightarrow^{w} x_{0}^{*}$ with $x_{n}^{*} \in \partial_{G}^{\pi} f\left(x_{n}\right)$. By the definition of $V$-p.l.n. functions, there are $\lambda_{1}, \lambda_{2}>0, c>0, T>0$ such that for any $t \geq T$, any $x \in \mathcal{N}_{V}\left(\bar{x}, \lambda_{1}, \lambda_{2}\right)$, and any $x^{*} \in \partial_{G}^{\pi} f(x) \cap c t \mathbb{B}_{*}$, we have:

$$
\left\langle x^{*} ; y-x\right\rangle \leq f(y)-f(x)+\frac{t}{2} V(J x, y), \quad \forall y \in \bar{x}+\lambda_{1} \mathbb{B} .
$$

For $n$ sufficiently large, we have $\left\|x_{n}^{*}\right\| \leq M$ and $x_{n} \in \mathcal{N}_{V}\left(\bar{x}, \lambda_{1}, \lambda_{2}\right)$. Let $t:=\max \left\{\frac{M}{c} ; T\right\}$. Obviously, $\left\|x_{n}^{*}\right\| \leq c t$ and $t \geq T$. Thus, for $n$ sufficiently large and any $y \in \bar{x}+\lambda_{1} \mathbb{B}$, we have:

$$
\left\langle x_{n}^{*} ; y-x_{n}\right\rangle \leq f(y)-f\left(x_{n}\right)+\frac{t}{2} V\left(J x_{n}, y\right), \quad \forall y \in \bar{x}+\lambda_{1} \mathbb{B} .
$$

By taking $n \rightarrow \infty$, we obtain:

$$
\left\langle x_{0}^{*} ; y-\bar{x}\right\rangle \leq f(y)-f(\bar{x})+\frac{t}{2} V(J \bar{x}, y), \quad \forall y \in \bar{x}+\lambda_{1} \mathbb{B},
$$

which means by definition that $x_{0}^{*} \in \partial^{\pi} f(\bar{x})$. Thus, $\partial_{G}^{L \pi} f(\bar{x}) \subset \partial^{\pi} f(\bar{x})$. Since the inclusions $\partial^{\pi} f(\bar{x}) \subset$ $\partial_{G}^{\pi} f(\bar{x}) \subset \partial_{G}^{L \pi} f(\bar{x})$ are always true, we deduce the desired equality $\partial_{G}^{L \pi} f(x)=\partial^{L \pi} f(x)=\partial^{\pi} f(x)=$ $\partial_{G}^{\pi} f(x)$. Furthermore, we always have $\partial^{\pi} f(\bar{x}) \subset \partial_{G}^{\pi} f(\bar{x}) \subset \partial^{F} f(\bar{x}) \subset \partial^{L F} f(\bar{x})$. Thus, $\partial^{L P} f(\bar{x})=$ $\partial^{L F} f(\bar{x})=\partial^{F} f(\bar{x})=\partial_{G}^{L \pi} f(\bar{x})=\partial^{L \pi} f(\bar{x})=\partial^{\pi} f(\bar{x})=\partial_{G}^{\pi} f(\bar{x})$, and the proof is complete.

Our next theorem shows the generalised $V$-prox-regularity of the epigraph for $V$-primal lower nice functions defined on $q$-uniformly convex and $p$-uniformly smooth, which is $V$-proximal trustworthy. First, we need to prove the following lemma.

Lemma 1. Let $X$ be a reflexive Banach space and $f: X \rightarrow \mathbb{R} \cup\{\infty\}$. For any $x \in \operatorname{dom} f$ and any $(x, \alpha) \in$ epif(i.e., $f(x) \leq \alpha$ ), we have:

$$
\left(x^{*}, 0\right) \in N^{\pi}(\text { epif } ;(x, \alpha)) \Longrightarrow\left(x^{*}, 0\right) \in N^{\pi}(\text { epif; }(x, f(x))) .
$$

Proof. Let $\left(x^{*}, 0\right) \in N^{\pi}$ (epi $\left.f ;(x, \alpha)\right)$. If $f(x)=\alpha$, then we are done. Assume now that $f(x)<\alpha$. Then, by (1), there exists $\rho>0$ such that:

$$
(x, \alpha)=\pi_{e p i f}\left(J x+\rho x^{*} ; \alpha+\rho \times 0\right)=\pi_{e p i f}\left(J x+\rho x^{*} ; \alpha\right),
$$

that is,

$$
V_{X \times \mathbb{R}}\left(\left(J x+\rho x^{*}, \alpha\right) ;(x, \alpha)\right)=\inf _{\left(y, \alpha^{\prime}\right) \in e p i f} V_{X \times \mathbb{R}}\left(\left(J x+\rho x^{*}, \alpha\right) ;\left(y, \alpha^{\prime}\right)\right)
$$

Hence,

$$
\begin{aligned}
V\left(J x+\rho x^{*} ; x\right) & =V\left(J x+\rho x^{*} ; x\right)+(\alpha-\alpha)^{2}=V_{X \times \mathbb{R}}\left(\left(J x+\rho x^{*}, \alpha\right) ;(x, \alpha)\right) \\
& =\inf _{\left(y, \alpha^{\prime}\right) \in e p i f} V_{X \times \mathbb{R}}\left(\left(J x+\rho x^{*}, \alpha\right) ;\left(y, \alpha^{\prime}\right)\right) \\
& =\inf _{\left(y, \alpha^{\prime}\right) \in e p i f}\left[V\left(J x+\rho x^{*} ; y\right)+\left(\alpha^{\prime}-\alpha\right)^{2}\right] \\
& \leq V\left(J x+\rho x^{*} ; y\right)+\left(\alpha^{\prime}-\alpha\right)^{2}, \quad \forall\left(y, \alpha^{\prime}\right) \in \text { epif. }
\end{aligned}
$$


Choose any $\delta \in(0, \alpha-f(x))$. Fix now any $\left(x^{\prime}, \alpha^{\prime}\right) \in$ epif with $\left(x^{\prime}, \alpha^{\prime}\right) \in(x, f(x))+\delta \mathbb{B}_{X \times \mathbb{R}}$, that is $\left\|x^{\prime}-x\right\|<\delta$ and $\left|\alpha^{\prime}-f(x)\right|<\delta$. We have:

$$
-\delta+\alpha^{\prime}<f(x)<\alpha^{\prime}+\delta \Longrightarrow \alpha^{\prime}<f(x)+\delta<\alpha \Longrightarrow f\left(x^{\prime}\right)<\alpha^{\prime}<\alpha,
$$

that is $\left(x^{\prime}, \alpha\right) \in$ epif. From (21), we have:

$$
V\left(J x+\rho x^{*} ; x\right) \leq V\left(J x+\rho x^{*} ; x^{\prime}\right)+(\alpha-\alpha)^{2}=V\left(J x+\rho x^{*} ; x^{\prime}\right) .
$$

Thus, for any $\left(x^{\prime}, \alpha^{\prime}\right) \in$ epi $f \cap\left[(x, f(x))+\delta \mathbb{B}_{X \times \mathbb{R}}\right]$, we have:

$$
V\left(J x+\rho x^{*} ; x\right)-V\left(J x+\rho x^{*} ; x^{\prime}\right) \leq 0 .
$$

On the other hand, we have by (3):

$$
V\left(J x+\rho x^{*} ; x\right)-V\left(J x+\rho x^{*} ; x^{\prime}\right)=-V\left(J x ; x^{\prime}\right)-2 \rho\left\langle x^{*} ; x-x^{\prime}\right\rangle .
$$

Therefore, for any $\left(x^{\prime}, \alpha^{\prime}\right) \in$ epi $f \cap\left[(x, f(x))+\delta \mathbb{B}_{X \times \mathbb{R}}\right]$, we obtain:

$$
\left\langle x^{*} ; x^{\prime}-x\right\rangle \leq \frac{1}{2 \rho} V\left(J x ; x^{\prime}\right),
$$

which ensures that:

$$
\begin{aligned}
\left.\left\langle\left(x^{*}, 0\right) ;\left(x^{\prime}, \alpha^{\prime}\right)-(x, f(x))\right)\right\rangle & \leq \frac{1}{2 \rho}\left[V\left(J x ; x^{\prime}\right)+\left[f(x)-\alpha^{\prime}\right]^{2}\right] \\
& =\frac{1}{2 \rho} V_{X \times \mathbb{R}}\left(\left(J_{X \times \mathbb{R}}(x, f(x)) ;\left(x^{\prime}, \alpha^{\prime}\right)\right) .\right.
\end{aligned}
$$

This ensures by (1) that $\left(x^{*}, 0\right) \in N^{\pi}($ epif $;(x, f(x)))$, and hence, the proof is complete.

Theorem 6. Let $X$ be a q-uniformly convex and p-uniformly smooth, which is $V$-proximal trustworthy. If $f$ is $V$-primal lower nice at $\bar{x} \in \operatorname{dom} f$, then epif is generalised $V$-prox-regular at $(\bar{x}, f(\bar{x}))$.

Proof. By the definition of $V$-p.l.n. at $\bar{x}$, we have positive numbers $\epsilon_{1} \in(0,1), \epsilon_{2}>0, r>0, T>0$ such that for any $t \geq T$, any $x \in \mathcal{N}_{V}\left(\bar{x}, \epsilon_{1}, \epsilon_{2}\right)$, and any $x^{*} \in \partial_{G}^{\pi} f(x) \cap r t \mathbb{B}_{*}$, we have:

$$
\left\langle x^{*} ; y-x\right\rangle \leq f(y)-f(x)+\frac{t}{2} V(J x, y), \quad \forall y \in \bar{x}+\epsilon_{1} \mathbb{B} .
$$

Choose $\epsilon_{0} \in\left(0, \frac{\epsilon_{1}}{2}\right)$ such that for any $z \in \bar{x}+\epsilon_{0} \mathbb{B}$, we have $V(J \bar{x}, z)<\epsilon_{2}$. This ensures the inclusion $\bar{x}+\epsilon_{0} \mathbb{B} \subset \mathcal{N}_{V}\left(\bar{x} ; \epsilon_{1}, \epsilon_{2}\right)$. Take $(x, \alpha) \in$ epi $f$ and $\left(x^{*},-\lambda\right) \in N^{\pi}$ (epi $\left.f ;(x, \alpha)\right) \cap \epsilon_{0} \mathbb{B}_{X^{*} \times \mathbb{R}}$ and $x \in$ $\bar{x}+\frac{\epsilon_{0}}{2} \mathbb{B}$ with $|f(\bar{x})-\alpha|<\frac{\epsilon_{0}}{2}$. Fix now any $\left(x^{\prime}, \alpha^{\prime}\right) \in$ epif with $\left\|x^{\prime}-\bar{x}\right\|<\epsilon_{0}$ and $\left|\alpha^{\prime}-f(\bar{x})\right|<\epsilon_{0}$. Clearly, $x^{\prime} \in \mathcal{N}_{V}\left(\bar{x} ; \epsilon_{1}, \epsilon_{2}\right)$.

Case 01: $\lambda>0$.

In this case, we necessarily have $\alpha=f(x)$. Then, $\left(\frac{x^{*}}{\lambda},-1\right) \in N^{\pi}($ epi $f ;(x, f(x))) \cap \epsilon_{0} \mathbb{B}_{X^{*} \times \mathbb{R}}$, so by definition of the geometric $V$-proximal subdifferential, we get $\frac{x^{*}}{\lambda} \in \partial_{G}^{\pi} f(x)$. Furthermore, we have $\left\|\frac{x^{*}}{\lambda}\right\| \leq \frac{\epsilon_{0}}{\lambda} \leq r t$ for every $t \geq \max \left\{T, \frac{1}{r \lambda}\right\}$. Hence, by (22), we obtain:

$$
\left\langle\lambda^{-1} x^{*} ; x^{\prime}-x\right\rangle \leq f\left(x^{\prime}\right)-f(x)+\frac{t}{2} V\left(J x, x^{\prime}\right),
$$


which entails:

$$
\begin{aligned}
\left\langle x^{*} ; x^{\prime}-x\right\rangle & \leq \lambda\left[f\left(x^{\prime}\right)-f(x)\right]+\frac{t \lambda}{2} V\left(J x, x^{\prime}\right) \\
& \leq \lambda\left[f\left(x^{\prime}\right)-f(x)\right]+\frac{t \lambda}{2} V\left(J x, x^{\prime}\right)+\frac{t \lambda}{2}\left[\alpha^{\prime}-\alpha\right]^{2}
\end{aligned}
$$

Since $f\left(x^{\prime}\right) \leq \alpha^{\prime}$, we have $\forall t^{\prime} \geq \max \left\{\frac{T}{2}, \frac{1}{2 r}\right\}$, and the following two inequalities hold:

$$
2 \lambda^{-1} t^{\prime} \geq \frac{1}{r \lambda} \quad \text { and } \quad 2 \lambda^{-1} t^{\prime} \geq \frac{T}{\lambda} \geq T ;
$$

hence, (23) entails with $t=2 \lambda^{-1} t^{\prime}$ :

$$
\begin{aligned}
\left\langle x^{*} ; x^{\prime}-x\right\rangle & \leq \lambda\left[f\left(x^{\prime}\right)-f(x)\right]+\left(2 \lambda^{-1} t^{\prime}\right) \frac{\lambda}{2} V\left(J x, x^{\prime}\right)+\left(2 \lambda^{-1} t^{\prime}\right) \frac{\lambda}{2}\left[\alpha^{\prime}-\alpha\right]^{2} \\
& \leq \lambda\left[f\left(x^{\prime}\right)-f(x)\right]+t^{\prime} V\left(J x, x^{\prime}\right)+t^{\prime}\left[\alpha^{\prime}-\alpha\right]^{2} \\
& \leq t^{\prime}\|x\|^{2}+t^{\prime}\left\|x^{\prime}\right\|^{2}-2 t^{\prime}\left\langle J x ; x^{\prime}-x\right\rangle+\lambda\left[\alpha^{\prime}-\alpha\right]+t^{\prime}\left[\alpha^{\prime}-\alpha\right]^{2} .
\end{aligned}
$$

Hence:

$$
\left\langle x^{*}+2 t^{\prime} J x ; x^{\prime}-x\right\rangle \leq t^{\prime}\left\|x^{\prime}\right\|^{2}-t^{\prime}\|x\|^{2}+\lambda\left[\alpha^{\prime}-\alpha\right]+t^{\prime}\left[\alpha^{\prime}-\alpha\right]^{2} .
$$

Therefore,

$$
t^{\prime}\|x\|^{2}-2 t^{\prime}\left\langle J x+\frac{x^{*}}{2 t^{\prime}} ; x\right\rangle \leq t^{\prime}\left\|x^{\prime}\right\|^{2}-2 t^{\prime}\left\langle J x+\frac{x^{*}}{2 t^{\prime}} ; x^{\prime}\right\rangle+\lambda\left[\alpha^{\prime}-\alpha\right]+t^{\prime}\left[\alpha^{\prime}-\alpha\right]^{2} .
$$

Dividing by $t^{\prime}>0$ yields:

$$
V\left(J x+\frac{x^{*}}{2 t^{\prime}} ; x\right) \leq V\left(J x+\frac{x^{*}}{2 t^{\prime}} ; x^{\prime}\right\rangle+\frac{\lambda}{t^{\prime}}\left[\alpha^{\prime}-\alpha\right]+\left[\alpha^{\prime}-\alpha\right]^{2} .
$$

On the other hand, we have:

$$
\begin{aligned}
{\left[\alpha^{\prime}-\alpha+\frac{1}{2 t^{\prime}} \lambda\right]^{2} } & =\left[\alpha^{\prime}-\alpha\right]^{2}+\frac{\lambda}{t^{\prime}}\left(\alpha^{\prime}-\alpha\right)+\frac{\lambda^{2}}{4 t^{\prime 2}} \\
& =\frac{1}{t^{\prime}}\left[t^{\prime}\left[\alpha^{\prime}-\alpha\right]^{2}+\lambda\left(\alpha^{\prime}-\alpha\right)+\frac{\lambda^{2}}{4 t^{\prime}}\right]
\end{aligned}
$$

Therefore,

$$
t^{\prime}\left[\alpha^{\prime}-\alpha+\frac{1}{2 t^{\prime}} \lambda\right]^{2}-\frac{\lambda^{2}}{4 t^{\prime}}=t^{\prime}\left[\alpha^{\prime}-\alpha\right]^{2}+\lambda\left(\alpha^{\prime}-\alpha\right) .
$$

Thus, (24) becomes:

$$
\begin{aligned}
V\left(J x+\frac{x^{*}}{2 t^{\prime}} ; x\right) & \leq V\left(J x+\frac{x^{*}}{2 t^{\prime}} ; x^{\prime}\right\rangle+\frac{1}{t^{\prime}}\left[t^{\prime}\left[\alpha^{\prime}-\alpha\right]^{2}+\lambda\left[\alpha^{\prime}-\alpha\right]\right] \\
& \leq V\left(J x+\frac{x^{*}}{2 t^{\prime}} ; x^{\prime}\right\rangle+\left[\alpha^{\prime}-\alpha+\frac{1}{2 t^{\prime}} \lambda\right]^{2}-\frac{\lambda^{2}}{4 t^{\prime 2}}
\end{aligned}
$$


and so:

$$
V\left(J x+\frac{x^{*}}{2 t^{\prime}} ; x\right)+\frac{\lambda^{2}}{4 t^{\prime 2}} \leq V\left(J x+\frac{x^{*}}{2 t^{\prime}} ; x^{\prime}\right\rangle+\left[\alpha^{\prime}-\alpha+\frac{1}{2 t^{\prime}} \lambda\right]^{2}
$$

Observe that:

$$
\begin{aligned}
V_{X \times \mathbb{R}}\left(\left(J x+\frac{x^{*}}{2 t^{\prime}},\left(\alpha+\frac{1}{2 t^{\prime}}(-\lambda)\right)\right) ;(x, \alpha)\right) & =V\left(J x+\frac{x^{*}}{2 t^{\prime}} ; x\right)+\frac{\lambda^{2}}{4 t^{\prime 2}}, \text { and } \\
V_{X \times \mathbb{R}}\left(\left(J x+\frac{x^{*}}{2 t^{\prime}},\left(\alpha+\frac{1}{2 t^{\prime}}(-\lambda)\right)\right) ;\left(x^{\prime}, \alpha^{\prime}\right)\right) & =V\left(J x+\frac{x^{*}}{2 t^{\prime}} ; x^{\prime}\right\rangle+\left[\alpha^{\prime}-\alpha+\frac{1}{2 t^{\prime}} \lambda\right]^{2} .
\end{aligned}
$$

Therefore,

$$
V_{X \times \mathbb{R}}\left(\left(J x+\frac{x^{*}}{2 t^{\prime}},\left(\alpha+\frac{1}{2 t^{\prime}}(-\lambda)\right)\right) ;(x, \alpha)\right) \leq V_{X \times \mathbb{R}}\left(\left(J x+\frac{x^{*}}{2 t^{\prime}}\left(\alpha+\frac{1}{2 t^{\prime}}(-\lambda)\right)\right) ;\left(x^{\prime}, \alpha^{\prime}\right)\right),
$$

for any $\left(x^{\prime}, \alpha^{\prime}\right) \in \operatorname{epi} f \cap\left[(\bar{x}, f(\bar{x}))+\epsilon_{0} \mathbb{B}_{X \times \mathbb{R}}\right]$, that is,

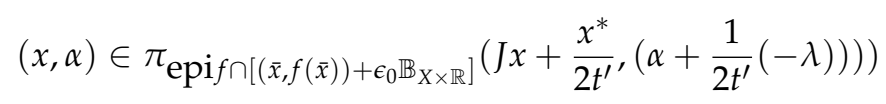

Case 02: $\lambda=0$.

In this case, we have $\left(x^{*} ; 0\right) \in N^{\pi}$ (epi $\left.f ;(x, \alpha)\right)$, and so, by Lemma 1 , we obtain $\left(x^{*}, 0\right) \in$ $N^{\pi}$ (epif; $\left.(x, f(x))\right)$. Using Proposition 2, there exist sequences $x_{n} \rightarrow x$ with $f\left(x_{n}\right) \rightarrow f(x), \mu_{n} \rightarrow 0^{+}$, and $y_{n}^{*} \in \mu_{n} \partial^{\pi} f\left(x_{n}\right)$ such that $\left\|y_{n}^{*}-x^{*}\right\|_{*} \rightarrow 0$. Let $x_{n}^{*}:=\mu_{n}^{-1} y_{n}^{*}$. Then, $\left\|\mu_{n} x_{n}^{*}-x^{*}\right\|=\left\|y_{n}^{*}-x^{*}\right\| \rightarrow$ 0 as $n \rightarrow \infty$, that is $\mu_{n} x_{n}^{*} \rightarrow x^{*}$. For $n$ large enough (i.e., $\forall n \geq N_{0}$ ), we have $\left\|x_{n}-x\right\|<\frac{\epsilon_{0}}{2}$ and $\mu_{n}<1$. Assume for a moment $x^{*} \neq 0$. Let $t_{n}:=\max \left\{\frac{T}{2 \mu_{n}}, \frac{\left\|x_{n}^{*}\right\|}{2 r\left\|x^{*}\right\|}\right\}, \forall n \geq N_{0}$, and we see that:

$$
2 t_{n} \geq \max \left\{T, \frac{\left\|x_{n}^{*}\right\|}{r}\right\}, \quad\left(\text { since } \frac{1}{\mu_{n}}>1 \text { and } \frac{1}{\left\|x^{*}\right\|}>1\right)
$$

and hence, by (22) with $y:=x^{\prime}, x:=x_{n}$, and $t:=2 t_{n}$, we obtain:

$$
\left\langle x_{n}^{*} ; x^{\prime}-x_{n}\right\rangle \leq f\left(x^{\prime}\right)-f\left(x_{n}\right)+t_{n} V\left(J x_{n}, x^{\prime}\right) .
$$

Multiplying this inequality by $\mu_{n}$, we get:

$$
\left\langle\mu_{n} x_{n}^{*} ; x^{\prime}-x_{n}\right\rangle \leq \mu_{n}\left[f\left(x^{\prime}\right)-f\left(x_{n}\right)\right]+\mu_{n} t_{n} V\left(J x_{n}, x^{\prime}\right) .
$$

Let $\rho_{n}:=\max \left\{\frac{T}{2}, \frac{\mu_{n}\left\|x_{n}^{*}\right\|}{2 r\left\|x^{*}\right\|}\right\}$. Clearly, $\rho_{n} \rightarrow \rho:=\max \left\{\frac{T}{2}, \frac{1}{2 r}\right\}$, and $t_{n} \mu_{n}=\rho_{n}$. Therefore, for any $n \geq N_{0}$, we have:

$$
\left\langle y_{n}^{*} ; x^{\prime}-x_{n}\right\rangle \leq \mu_{n}\left[f\left(x^{\prime}\right)-f\left(x_{n}\right)\right]+\rho_{n} V\left(J x_{n}, x^{\prime}\right) .
$$

Now, taking the limit as $n \rightarrow \infty$ yields by the continuity of $V$ and $J$ :

$$
\left\langle x^{*} ; x^{\prime}-x\right\rangle \leq \rho V\left(J x, x^{\prime}\right) \leq \rho V\left(J x, x^{\prime}\right)+\rho\left[\alpha^{\prime}-\alpha\right]^{2}
$$

Therefore:

$$
\left\langle x^{*} ; x^{\prime}\right\rangle \leq\left\langle x^{*} ; x\right\rangle+\rho\|x\|^{2}+\rho\left\|x^{\prime}\right\|^{2}-2 \rho\left\langle J x ; x^{\prime}\right\rangle+\rho\left[\alpha^{\prime}-\alpha\right]^{2} .
$$

Thus,

$$
\rho\|x\|^{2}-\left\langle x^{*}+2 \rho J x ; x\right\rangle \leq \rho\left\|x^{\prime}\right\|^{2}-\left\langle x^{*}+2 \rho J x ; x^{\prime}\right\rangle+\rho\left[\alpha^{\prime}-\alpha\right]^{2} .
$$


Dividing by $\rho>0$ gives:

$$
\|x\|^{2}-2\left\langle J x+\frac{1}{2 \rho} x^{*} ; x\right\rangle \leq\left\|x^{\prime}\right\|^{2}-2\left\langle J x+\frac{1}{2 \rho} x^{*} ; x^{\prime}\right\rangle+\left[\alpha^{\prime}-\alpha\right]^{2}
$$

Therefore:

$$
V\left(J x+\frac{1}{2 \rho} x^{*}, x\right) \leq V\left(J x+\frac{1}{2 \rho} x^{*}, x^{\prime}\right)+\left[\alpha^{\prime}-\alpha\right]^{2} .
$$

This ensures for $\lambda=0$ :

$$
V_{X \times \mathbb{R}}\left(\left(J x+\frac{x^{*}}{2 \rho},\left(\alpha+\frac{1}{2 \rho}(-\lambda)\right)\right) ;(x, \alpha)\right) \leq V_{X \times \mathbb{R}}\left(\left(J x+\frac{x^{*}}{2 \rho},\left(\alpha+\frac{1}{2 \rho}(-\lambda)\right)\right) ;\left(x^{\prime}, \alpha^{\prime}\right)\right),
$$

for any $\left(x^{\prime}, \alpha^{\prime}\right) \in \operatorname{epi} f \cap\left[(\bar{x}, f(\bar{x}))+\epsilon_{0} \mathbb{B}_{X \times \mathbb{R}}\right]$, that is,

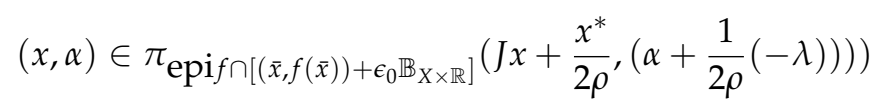

Finally, we obtain from Case 01 and Case 02 two positive numbers $\epsilon_{0}$ and $r_{0}:=\frac{1}{2 \rho}$ such that for any $(x, \alpha) \in$ epi $f$ and $\left(x^{*},-\lambda\right) \in N^{\pi}($ epif $;(x, \alpha)) \cap \epsilon_{0} \mathbb{B}_{X \times \mathbb{R}}$ and any $x \in \bar{x}+\epsilon_{0} \mathbb{B}$, and $|f(\bar{x})-\alpha|<\epsilon_{0}$, we have:

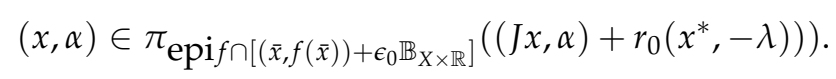

This means by Definition 3 that the epigraph epi $f$ is generalised $V$-prox-regular at $(\bar{x}, f(\bar{x}))$, and hence, the proof is complete.

Author Contributions: Investigation, M.B. (Messaoud Bounkhel) and M.B. (Mostafa Bachar). All authors contributed equally to this article. All authors read and agreed to the published version of the manuscript.

Funding: The authors extend their appreciation to the Deanship of Scientific Research at King Saud University for funding the work through the research group Project No. RGP-024.

Acknowledgments: The authors would like to thank the three referees for their valuable remarks and suggestions.

Conflicts of Interest: The authors declare no conflict of interest.

\section{References}

1. Bounkhel, M.; Al-Yusof, R. Proximal analysis in reflexive smooth Banach spaces. Nonlinear Anal. 2010, 73, 1921-1939. [CrossRef]

2. Alber, Y. Generalised projection operators in Banach spaces: Properties and applications. Funct. Differ. Equ. 1994, 1, 1-21.

3. Bounkhel, M. Generalised projections on closed nonconvex sets in uniformly convex and uniformly smooth Banach spaces. J. Funct. Spaces 2015, 2015. [CrossRef]

4. Bounkhel, M. Regularity Concepts in Nonsmooth Analysis, Theory and Applications; Springer: Berlin/Heidelberg, Germany, 2012; Volume 59.

5. Bounkhel, M.; Bachar, M. V-proximal trustworthy Banach spaces. J. Funct. Spaces 2020, 2020, 1-5. [CrossRef]

6. Bounkhel, M. Calculus rules for $V$-proximal subdifferentials in smooth Banach spaces. J. Funct. Spaces 2016, 2016. [CrossRef]

7. Bounkhel, M.; Bachar, M. Generalized prox-regularity in reflexive Banach spaces. J. Math. Anal. Appl. 2019, 475, 699-729. [CrossRef]

8. Levy, A.; Polquin, R.; Thibault, L. Partial extension of Attouch's theorem with applications to proto-derivatives on subgradient mappings. Trans. Am. Math. Soc. 1995, 347, 1269-1294.

9. Poliquin, R. Integration of subdifferentials of nonconvex functions. Nonlinear Anal. 1991, 17, $385-398$. [CrossRef]

10. Ivanov, M.; Zlateva, N. On primal lower nice property. Comptes Rendus l'Académie Bulg. Sci. 2001, 54, 5-10.

11. Combari, C.; Elhilali Alaoui, A.; Levy, A.; Poliquin, R.; Thibault, L. Convex composite functions in Banach spaces and the primal lower-nice property. Proc. Am. Math. Soc. 1998, 126, 3701-3708. [CrossRef] 
12. Thibault, L.; Zagrodny, D. Integration of subdifferentials of lower semicontinuous functions on Banach spaces. J. Math. Anal. Appl. 1995, 189, 33-58. [CrossRef]

Publisher's Note: MDPI stays neutral with regard to jurisdictional claims in published maps and institutional affiliations.

(C) 2020 by the authors. Licensee MDPI, Basel, Switzerland. This article is an open access article distributed under the terms and conditions of the Creative Commons Attribution (CC BY) license (http://creativecommons.org/licenses/by/4.0/). 